\title{
Multidirectional Transparent Support for Overground Gait Training
}

\author{
H. Vallery* ${ }^{* \dagger}$, P. Lutz ${ }^{\S}$, J. von Zitzewitz ${ }^{\dagger}$, G. Rauter ${ }^{\dagger} \|$, M. Fritschi ${ }^{\ddagger}$, \\ C. Everarts ${ }^{\ddagger \ddagger}$, R. Ronsse ${ }^{\ddagger \ddagger}$, A. Curtll and M. Bolliger $\|$ \\ *Delft University of Technology, 2628 CD Delft, The Netherlands, h.vallery@tudelft.nl \\ †Sensory-Motor Systems Lab, ETH Zurich, 8092 Zurich, Switzerland \\ $\ddagger$ Khalifa University of Science, Technology and Research, Abu Dhabi, UAE \\ $\S$ Lutz Medical Engineering, 8455 Rüdlingen, Switzerland \\ ICenter for Neuroprosthetics and Brain Mind Institute, EPFL Lausanne, 1015 Lausanne, Switzerland \\ $\ddagger$ Center for Research in Mechatronics, Université catholique de Louvain, 1348 Louvain-la-Neuve, Belgium \\ " Spinal Cord Injury Center, University of Zurich, Balgrist University Hospital, Zurich, Switzerland
}

\begin{abstract}
Gait and balance training is an essential ingredient for locomotor rehabilitation of patients with neurological impairments. Robotic overhead support systems may help these patients train, for example by relieving them of part of their body weight. However, there are only very few systems that provide support during overground gait, and these suffer from limited degrees of freedom and/or undesired interaction forces due to uncompensated robot dynamics, namely inertia. Here, we suggest a novel mechanical concept that is based on cable robot technology and that allows three-dimensional gait training while reducing apparent robot dynamics to a minimum. The solution does not suffer from the conventional drawback of cable robots, which is a limited workspace. Instead, displaceable deflection units follow the human subject above a large walking area. These deflection units are not actuated, instead they are implicitly displaced by means of the forces in the cables they deflect. This leads to an underactuated design, because the deflection units cannot be moved arbitrarily. However, the design still allows accurate control of a three-dimensional force vector acting on a human subject during gait. We describe the mechanical concept, the control concept, and we show first experimental results obtained with the device, including the force control performance during robot-supported overground gait of five human subjects without motor impairments.
\end{abstract}

Keywords-Gait training, body weight support, cable robots, series elastic actuation, underactuation.

\section{INTRODUCTION}

A growing body of clinical studies suggests that effective training in neurorehabilitation allows subjects to participate actively and to perform unhindered movements. Therefore, strategies like "Assist as Needed" [1] or the "challenge point" concept [2] recommend tailor-made assistance or challenge during training. For gait rehabilitation robots, this denotes a change in paradigm, because currently available devices have been designed to ensure accurate reproduction of physiological kinematics. Such devices cannot fully "get out of the way" when not needed, because their intrinsic dynamics generate undesired interaction forces.

One step towards more cooperative training environments was marked by the "KineAssist" robot [3], which is a moving frame with attached harness that can gently catch the subject in case of fall. However, the device cannot move fast due to its heavy structure, so it is more suitable for use in standing and stepping training, when the robot does not need to move.

We recently suggested a multidirectional overhead support system for rats [4], which allows highly transparent gait training. With this device, it was shown that treadmill training does not necessarily promote voluntary gait [5], in contrast to body-weight supported overground training. However, the mechanical structure of this robot cannot simply be enlarged to human scale.

Classical body weight support (BWS) systems for humans are often realized by use of cable systems. Mostly the user wears a harness, which is suspended by a single cable. The cable is tensed by passive and/or active elements, resulting in a 1-dimensional, uni-directional vertical force. Adding further dimensions is often realized by repositioning the pulley guiding the cable to the user. For example, the Zero-G [6] provides support during overground walking by means of a trolley that runs on a rail and contains a pulley mechanism. However, this constrains subjects to walking along a given path, which unfavorably influences balance tasks by generating horizontal "pendulum" forces [7].

NASA's ARGOS [8] or the NaviGAITor [9] are overhead gantry systems that also allow lateral movements. However, their massive structure leads to low bandwidth and restricts dynamic movements of the subject.

Another approach for multi-directional support is represented by cable-driven robots [10], [11]: Spatially distributed pulleys guide multiple cables, which are driven by motorized drums, into the workspace. The free cable ends inside the workspace are attached in parallel to one or multiple points. Such cable robots, as parallel robots in general, usually have one disadvantage: Their load capabilities vary strongly depending on the position of the end-effector inside the workspace. This results from the increasingly inhomogeneous distribution of cable force vectors when the end-effector is moved away from its central position towards the outer workspace zones. This disadvantage can be compensated by actively moving the pulleys; however, this solution is costly due to its high mechanical complexity [12]. 
Here, we suggest a new concept for a cable robot for gait and balance training that employs pulleys that are neither fixed nor actuated. Instead, they are moved along guiding rails by the forces resulting through the deflection of the cables while being directed to the human subject [13], [12]. This general concept minimizes moving masses of the robot. This way, we achieve minimal inertia, yet still a large workspace. As a consequence of the passive deflection units, the device is underactuated [14]. However, appropriate control still allows exact control of the forces that are exerted on the user.

In the following sections, we will first describe the hardware and control concept, followed by an experimental evaluation that identifies apparent robot dynamics and that assesses force control performance in experiments with human subjects.

\section{HARDWARE CONCEPT}

\section{A. Mechanics}

The robotic system FLOAT (Free Levitation for Overground Active Training) is an overhead support system that is designed to precisely control forces acting on a human subject in vertical and in both horizontal directions (Fig.1). The device capitalizes on cable robot technology.

The FLOAT allows the subjects to move in a large space (approximately $8 \mathrm{~m}$ length by $1.5 \mathrm{~m}$ width by $2.8 \mathrm{~m}$ height in our setup in Zürich, which is mainly limited by room size), so that diverse activities can be trained and analyzed in healthy subjects or in patients, such as level walking, running, walking on uneven terrain, and even stair climbing. During these activities, FLOAT needs to transparently follow the subject while precisely controlling magnitude and direction of the force vector acting on the human subject. To this end, two parallel rails are arranged horizontally on the ceiling and tilted by $45^{\circ}$ towards the workspace along their longitudinal axis. Each rail guides two deflection units: Each deflection unit is composed of a ball-beared cart carrying an inclinable pulley. The inclination axis of the pulley is parallel to the rail. A Dyneema cable with $2.5 \mathrm{~mm}$ diameter connects the two carts on one rail, so that they form a "trolley".

At each end of the two rails, a winch is positioned. From each winch, a cable extends via the closest deflection unit into the workspace. The design of the winches (Fig. 1, inset right) is similar to the one proposed in [15]. AC motors (type AKM54G from Kollmorgen) with $2.6 \mathrm{~kW}$ power, a continuous torque of $14.3 \mathrm{Nm}$, and with an integrated brake are used. The motor shaft is connected to the drum via a backlash-free shaft coupling. The Polyamid drums have an effective diameter of $71 \mathrm{~mm}$ and a length of $245 \mathrm{~mm}$. The entire drive train (i.e. motor shaft with brake, coupling, shaft within the drum, drum) has a moment of inertia of $0.00186 \mathrm{kgm}^{2}$. Each winch unit is additionally equipped with a light-weight hollow pressure roller, which maintains the contact pressure of the cable on the drum in order to avoid derailing of the cable. The hollow roller rotates passively and its contact pressure can be adjusted. The cables are Dyneema ropes with $4 \mathrm{~mm}$ diameter.

Following the principle of Series Elastic Actuation [16], [17], four elastic elements connect the cables to stainless steel rings, which are arranged so that the four cables approximately intersect in one point, which we define as the "node" (Fig. 2).
This virtual intersection of the cables is located in the center of a steel ring. The elastic elements consist of spiral steel springs, each with a parallel rubber cord inside. They exhibit a slightly nonlinear force-length relationship, with a mean stiffness of approximately $6.2 \mathrm{kN} / \mathrm{m}$.

The human user wears a harness, which is attached to a beam, which in turn is pivot-mounted to the lower end of the node. Due to these decoupling joints, the steel ring in the node always aligns in direction of the resultant force vector that is generated by the four cables and acts on the subject. The beam can rotate infinitely, allowing the subject to take arbitrary turns. The beam further includes an emergency release system, allowing the subject to be lowered manually in case of electronic and/or mechanical failure of the robot.

In this setup, the force vector acting on the human can theoretically assume any direction within the polygon that is defined by five vertices: the node and the four winches (or, more precisely, extreme locations of the deflection units). This polygon is much larger than the one spanned by the node and any current location of the deflection units, which would confine the force vector in case the pulleys were fixed.

\section{B. Sensing, Communication, and Safety}

Winch positions, and thereby the amount of cable that has been released from the winch, are measured by multi-turn high-resolution encoders on the motor shafts.

The lengths of the elastic elements and thereby the force acting on any single cable is measured by wire potentiometers (Micro-Epsilon, Ortenburg) guided inside the spiral spring, next to the rubber cord.

The position of each of the carts is measured by a laser distance sensor, which is attached next to the respective winch. The laser beams are collinear to the rails. As there are two laser sensors per trolley (one for each cart), the measurement is redundant and is used both to improve signal quality and to serve for fault detection purposes.

These sensors already provide redundant information to calculate the position of the node and the resultant force vector on the subject by means of optimization, similar to [18].

Additionally, inside the steel ring in the node, an inertial measurement unit (IMU) is located, which contains accelerometers, gyroscopes, and a magnetometer (Fig. 2). This unit measures accelerations, angular rates, and orientation of the steel ring in the node in 3D. As the steel ring always aligns with the resultant force acting on the user, the IMU unit senses the direction of this force. The orientation is redundant to the information from cable forces and geometry, so that it allows improvement of signal quality as well as fault detection. In addition, the IMU can be used to estimate speed and acceleration of the node, which is beneficial for dynamic control regimes. In the current state of development, however, the IMU is not yet used in the control scheme.

Below the node, a rotary potentiometer is attached, which measures the rotation of the harness beam around the vertical axis, and thereby the current walking direction of the user.

The signals from the IMU and the five potentiometers are transmitted the control unit via a spiral cable attached to an 


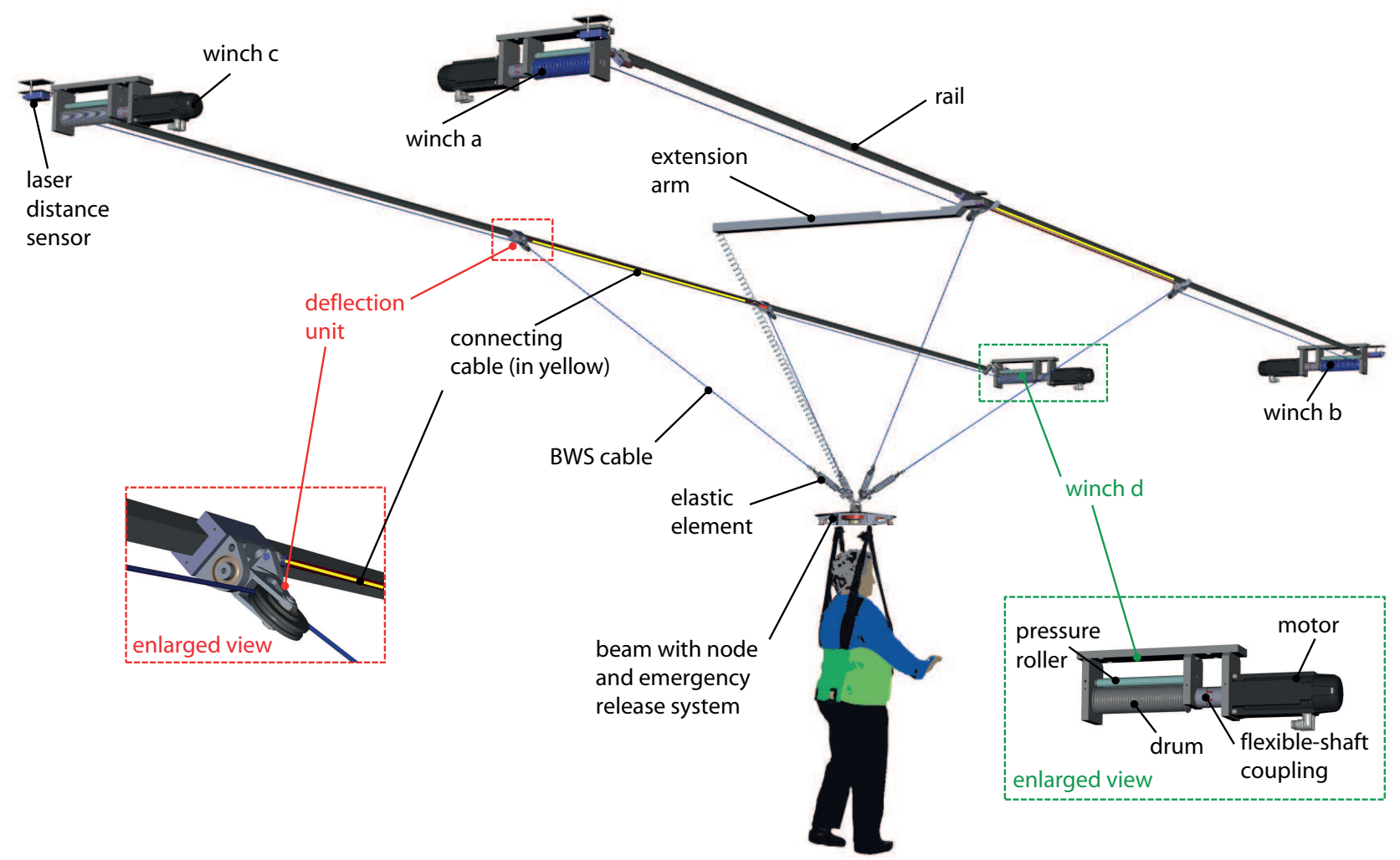

Fig. 1. Concept of the FLOAT training system, with enlarged views of a deflection unit and a winch unit.

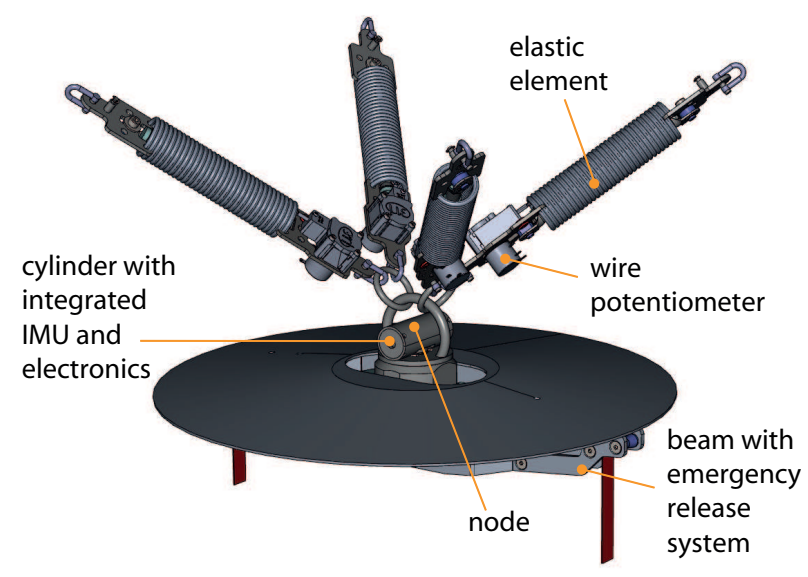

Fig. 2. Detailed view of elastic elements and node.

extension arm. The extension arm is mounted to a fifth cart, which is driven by a dedicated motor via a belt. The position of this cart is measured by an additional laser sensor, in order to enable a position controller that keeps the distance to the neighboring trolley constant.

Communication is realized with an updated and extended version of the solution proposed in [19]: The real-time control PC (Matlab xPC) and the motor drives communicate via an EtherCat network. Also the signals of the laser sensor are digitized by decentral EtherCat boxes (Beckhoff, Germany) and transmitted over this network. The EtherCat cycle and the control algorithms run at a frequency of $1 \mathrm{kHz}$. The signals that are collected at the node (lengths of wire potentiometers, angle of rotary potentiometers, and signals of the IMU) are digitized (in case of the analog wire potentiometers) and transmitted via RS485 to the xPC (Fig. 3).

In addition to diverse software fault detection mechanisms, a human operator can halt the FLOAT anytime by a wireless emergency stop or two wall-mounted emergency stops. A watchdog surveys the operational reliability of the xPC-target, by verifying the correct generation of a pulsed check signal. The signals from the watchdog, emergency switches, remote control, motor drives, and the xPC-target are all surveyed by a FLEXI soft safety control unit (SICK AG, Stans, Switzerland), which enables/disables the motor drives and the main power supply.

\section{Modeling AND CONTROL}

\section{A. Modeling}

In the chosen right-handed Cartesian coordinate system, $z$ points upward and $x$ points forward in the default gait direction, parallel to the rails (Fig. 4). As the joints in the node ensure that only a force vector is transmitted, the harness can be represented by a single cable that connects the node $\boldsymbol{n}=\left(\begin{array}{lll}n_{x} & n_{y} & n_{z}\end{array}\right)^{T}$ to a specific point on the human.

The geometric configuration is fully described by the length of cables that have been released from each winch, subsumed in the vector $\boldsymbol{s}_{W} \in \mathbb{R}^{4}$, with $\boldsymbol{s}_{W}=\left(\begin{array}{llll}s_{a} & s_{b} & s_{c} & s_{d}\end{array}\right)^{T}$, and by the positions of the trolleys, subsumed in the vector $\boldsymbol{x}_{T}=$ $\left(\begin{array}{ll}x_{T, a b} & x_{T, c d}\end{array}\right)^{T}$. This means that a twelve-dimensional vector (containing $\boldsymbol{s}_{W}, \boldsymbol{x}_{T}, \dot{\boldsymbol{s}}_{W}$, and $\dot{\boldsymbol{x}}_{T}$ ) suffices to describe the current state of the robot. 


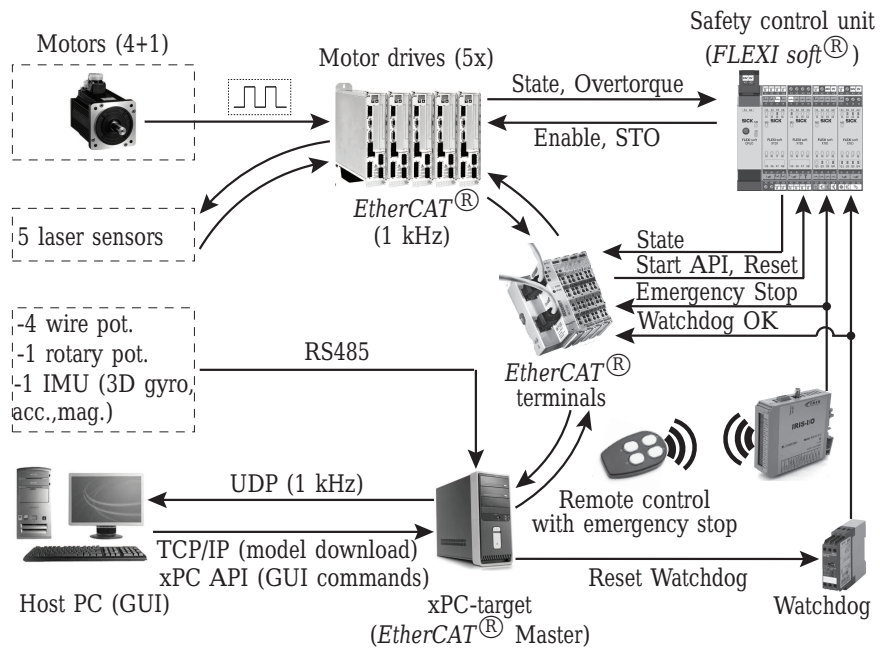

Fig. 3. Communication and safety flow chart of the FLOAT system.

Cable forces are subsumed in the vector $\boldsymbol{F}_{r} \in \mathbb{R}_{+}^{4}$ with $\boldsymbol{F}_{r}=\left(\begin{array}{lllll}F_{a} & F_{b} & F_{c} & F_{d}\end{array}\right)^{T}$, and the Cartesian force vector $\boldsymbol{F}_{n} \in \mathbb{R}^{3}$ acting on the subject is $\boldsymbol{F}_{n}=\left(\begin{array}{lll}F_{n x} & F_{n y} & F_{n z}\end{array}\right)^{T}$. Force equilibrium on the node maps cable forces to node forces:

$$
\boldsymbol{F}_{n}=\mathbf{J}\left(\boldsymbol{x}_{T}, \boldsymbol{n}\right) \boldsymbol{F}_{r}
$$

The Jacobian $\mathbf{J}$ can be computed in an efficient way by first summing up the cable forces within the two planes spanned by the cables, via the matrix $\mathbf{R}$, to obtain the component $F_{n, x}$ and the components $F_{a b}$ and $F_{c d}$ (Fig. 4, right), and then converting these to Cartesian space via the matrix $\mathbf{S}$ :

$$
\mathbf{J}=\left(\begin{array}{ll}
1 & \mathbf{0} \\
\mathbf{0} & \mathbf{S}
\end{array}\right) \mathbf{R}
$$

with

$$
\begin{aligned}
\mathbf{S}= & \left(\begin{array}{cc}
-\cos \varphi_{a b} & \cos \varphi_{c d} \\
\sin \varphi_{a b} & \sin \varphi_{c d}
\end{array}\right) \\
\mathbf{R}= & \left(\begin{array}{cccc}
\cos \varphi_{a} & -\cos \varphi_{b} & \cos \varphi_{c} & -\cos \varphi_{d} \\
\sin \varphi_{a} & \sin \varphi_{b} & 0 & 0 \\
0 & 0 & \sin \varphi_{c} & \sin \varphi_{d}
\end{array}\right) .
\end{aligned}
$$

Current trolley positions $\boldsymbol{x}_{T}$ and the node position $\boldsymbol{n}$ define the angles in these matrices (Fig. 4).

Trolley movement is governed by the equations of motion:

$$
m_{T} \ddot{\boldsymbol{x}}_{\boldsymbol{T}}=\mathbf{T} \boldsymbol{F}_{r},
$$

with

$$
\mathbf{T}=\left(\begin{array}{cccc}
\cos \varphi_{a}-1 & 1-\cos \varphi_{b} & 0 & 0 \\
0 & 0 & \cos \varphi_{c}-1 & 1-\cos \varphi_{d}
\end{array}\right)
$$

The equations of motion for the winches are given by:

$$
m_{W} \ddot{\boldsymbol{s}}_{W}=\boldsymbol{F}_{r}-\boldsymbol{F}_{W},
$$

with the winch actuator forces $\boldsymbol{F}_{W}$. This means that in static conditions or when the winches rotate at a constant speed, cable forces are equal to winch forces.

\section{B. Control Design}

An ideal controller would command actuator torques in such a way that the force vector $\boldsymbol{F}_{n}$ that acts on the subject matches the reference force vector $\boldsymbol{F}_{n, \text { ref }}$ regardless of the movement of the subject.

We implement a force controller in Cartesian space, which commands a Cartesian force vector ${ }^{C} \boldsymbol{F}_{f c}$ that is to be realized by the winches. This force is calculated by PI control and feedforward of the reference:

$$
{ }^{C} \boldsymbol{F}_{f c}=\boldsymbol{F}_{n, r e f}+\left(\mathbf{K}_{P}+\frac{\mathbf{K}_{I}}{s}\right)\left(\boldsymbol{F}_{n, r e f}-\boldsymbol{F}_{n}\right),
$$

with $s$ being the Laplace operator, $\mathbf{K}_{P}$ being a positive definite matrix of proportional gains, and $\mathbf{K}_{I}$ being a positive definite matrix of integral gains.

Cartesian forces need to be mapped to winch forces $\boldsymbol{F}_{w}$, which is the inverse problem of (1). Given that there are four winch forces and only three node force components, there are multiple solutions to (1) with a given node force. If the trolleys were not movable, quadratic programming could be used to find the minimal cable forces that fulfill the constraints. However, in the current system, the cable forces do not only influence the output force vector, but they also influence the movement of the trolleys, according to (5). In turn, the position of the trolleys defines the polygon of applicable forces.

Therefore, instead of minimizing cable forces, we take trolley dynamics into account to solve the rank deficiency in the inverse mapping of (1). The idea is to generate cable forces that force the trolleys to stay together, leading to a polygon with rectangular base. This behavior is enforced by the law:

$$
m_{T}\left(\ddot{x}_{T, a b}-\ddot{x}_{T, c d}\right) \stackrel{!}{=}-k_{T}\left(x_{T, a b}-x_{T, c d}\right)
$$

with the positive constant $k_{T}$.

With (5) and (6), this gives

$$
\boldsymbol{r}^{T} \boldsymbol{F}_{r} \stackrel{!}{=} k_{T}\left(x_{T, a b}-x_{T, c d}\right),
$$

whereby

$$
\boldsymbol{r}^{T}=\left(\begin{array}{llll}
1-\cos \varphi_{a} & \cos \varphi_{b}-1 & \cos \varphi_{c}-1 & 1-\cos \varphi_{d}
\end{array}\right) .
$$

Using this additional control goal, the control law maps reference forces in Cartesian space to winch forces:

$$
\boldsymbol{F}_{w}=\mathbf{R}^{\prime-1}\left(\begin{array}{cc}
\left(\begin{array}{cc}
1 & \mathbf{0} \\
\mathbf{0} & \mathbf{S}^{-1}
\end{array}\right){ }^{C} \boldsymbol{F}_{f c} \\
k_{T}\left(x_{T, a b}-x_{T, c d}\right)
\end{array}\right)
$$

with the reference force in Cartesian space $\boldsymbol{F}_{n, r e f}$ and the modified mapping matrix

$$
\mathbf{R}^{\prime}=\left(\begin{array}{c}
\mathbf{R} \\
\boldsymbol{r}^{T}
\end{array}\right)
$$

The winch forces are realized via underlying control loops, which also realize substantial artificial damping based on the winch velocity, similar to [20]. 

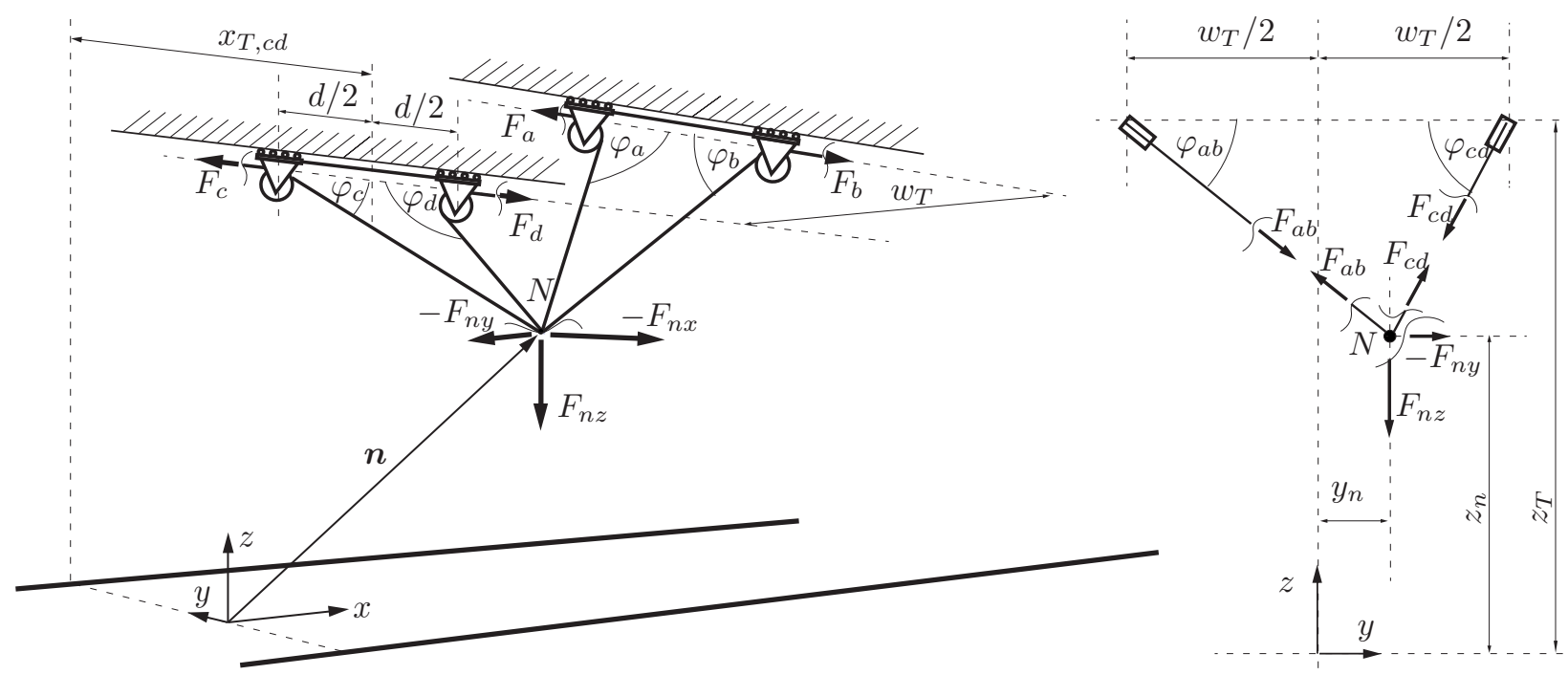

Fig. 4. Free-Body Diagrams with coordinate and variable definitions; 3D view and projection into $y-z$-plane. The object or human subject is connected to the node $N$. The force $\boldsymbol{F}_{n}$ acts on the subject, while the reaction force $-\boldsymbol{F}_{n}$ acts on the robot.

\section{EVALUATION PROTOCOL}

\section{A. Identification of Apparent Dynamics}

In a first experiment, we aimed to identify apparent robot dynamics by exciting the node in all three directions. For each direction individually, we modeled the robot as a linear mass-damper system, whose uncompensated dynamics can be captured by a simple linear model:

$$
\Delta \hat{F}_{i}=m \ddot{n}_{i}+d \dot{n}_{i} \text {, with } i \in\{x, y, z\} .
$$

The parameters $m$ and $d$ are the model's mass and damping factor, and $\Delta \hat{F}_{i}$ is the predicted difference between reference and actual force (as this is only the difference, gravitational components do not occur in the equation).

In order to identify the two parameters, harmonic movements at different frequencies were manually applied to the node, separately for each direction. During these experiments, the robot tracked a reference force in vertical direction of $200 \mathrm{~N}$, to maintain cable tension.

For the individual data sets for each direction, we found $m$ and $d$ by minimizing the linear-quadratic cost function

$$
J=\left|\left(F_{r e f, i}-F_{n, i}\right)-\Delta \hat{F}_{i}\right|^{2}, i \in\{x, y, z\} .
$$

Values for velocity and acceleration of the node were calculated via offline differentiation of the low-pass-filtered node position (non-causal 4th-order Butterworth with cutoff frequency $10 \mathrm{~Hz}$ ).

As the elastic elements measure the force that acts on the node, not directly on the user, the identified mass has to be augmented by the mass of the beam and harness structure, which is approximately $5 \mathrm{~kg}$. This mass is mainly caused by the emergency release system.

To validate the model, we recorded an additional data set where all directions were excited simultaneously, to assess cross-talk effects. To compare this data with the model predictions, we calculated the coefficient of determination $R^{2}$.

\section{B. Force Control Performance during Walking}

In a second experiment, we aimed to quantify force control performance in realistic conditions. Five subjects $(2 \mathrm{~m} / 3 \mathrm{f}$, aged $25-30 \mathrm{y}$, weight $50-85 \mathrm{~kg}$ ) with no known movement disorders participated. Subjects were asked to walk back and forth while attached to the robot and receiving constant body weight support for a duration of $30 \mathrm{~s}$.

The controller described in Sec. III-B was used to track a constant reference force in vertical direction, and zero forces in horizontal directions, as long as the subject remained within virtual workspace limits. At the workspace extremities, an additional spring-like force was applied to limit the workspace before reaching the actual mechanical limits. Subjects were allowed to use the haptic information from these "virtual walls", especially in $x$ direction, to know when to stop and turn around. The experiment was done with two different BWS values in $z$-direction: $10 \mathrm{~kg}$ and $25 \mathrm{~kg}$. As the beam and harness have an additional mass of $5 \mathrm{~kg}$, the reference force in $z$ direction was increased by an equivalent amount.

Off-line, the acquired force data was smoothed with a 4th-order phaseless Butterworth low-pass filter with a cut-off frequency of $50 \mathrm{~Hz}$. Force tracking performance was evaluated with the root mean square (RMS) error between the reference and the actual force:

$$
R M S_{i}=\sqrt{\frac{1}{N} \sum_{k=1}^{N}\left[F_{n, i}(k)-F_{r e f, i}\right]^{2}}, i \in\{x, y, z\} .
$$

where $N$ is the number of registered samples.

To assess the impact of these forces on human gait, we also recorded the displacement of the node in the three directions, which approximately reflects the movement of the subject's center of mass. From this data, we also calculated the node speed in walking direction, by numerical differentiation with a $10 \mathrm{~Hz}$, non-causal 4th-order Butterworth low-pass filter. 


\section{RESUlts}

\section{A. Apparent Dynamics}

The identified values for the apparent mass $m$ were $0.00 \mathrm{~kg}$ in $x$-direction, $1.04 \mathrm{~kg}$ in $y$-direction, and $1.02 \mathrm{~kg}$ in $z$-direction, and the values for damping $d$ were $9.7 \mathrm{Ns} / \mathrm{m}$, $67.4 \mathrm{Ns} / \mathrm{m}$, and $61.6 \mathrm{Ns} / \mathrm{m}$ for $x, y$, and $z$, respectively. The model matched the multidimensional evaluation data with a coefficient of determination $R^{2}$ of 0.68 for $x, 0.94$ for $y$, and 0.91 for $z$. An excerpt of the evaluation data and the model predictions is depicted in (Fig. 5). In all directions, the additional (uncompensable) mass of the bar and harness construction further adds to the apparent mass (with $5 \mathrm{~kg}$ ).

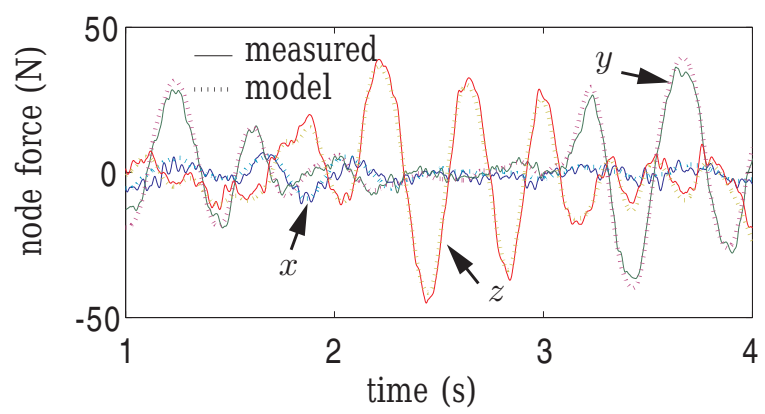

Fig. 5. Measured and predicted forces for multi-directional excitation.

\section{B. Force Control Performance during Walking}

Fig. 6, Fig. 7, and Fig. 8 show a concatenation of tensecond excerpts from each subject with $10 \mathrm{~kg}$ BWS.
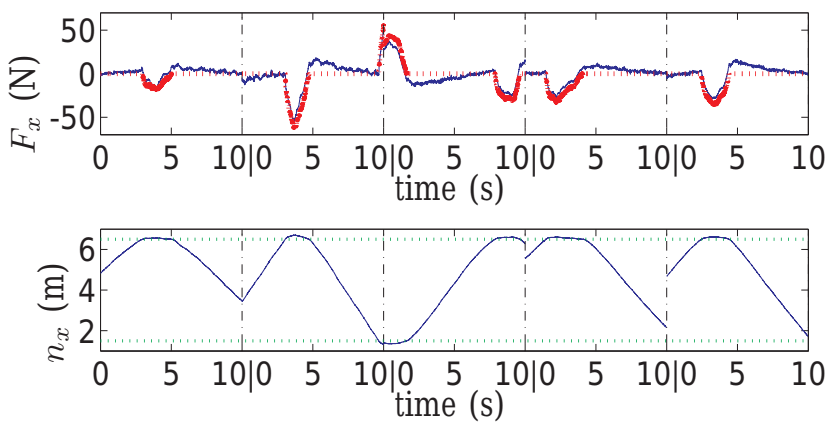

Fig. 6. Concatenated data excerpts from the 5 subjects in $x$-direction Top: Reference force (dotted red) and measured force (solid blue). Bottom Associated node movement in $x$-direction, with virtual walls (dotted green).

The node forces that were measured are shown in the top plots of the figures. The reference force in the $x$ and $y$ directions was mostly zero, unless the subjects hit the virtual workspace boundaries. This mostly happened in $x$-direction. In the $z$-direction, the boundaries were not reached because no large vertical movements were performed in this direction.

Periodic force fluctuations in response to human movements are observed in all three directions (the ones in $x$ are less visible, due to a scaling effect). Across all data sets with the full 30 seconds for each subject, the RMS error was calculated: For $10 \mathrm{~kg}$ BWS, the RMS error in force was $6.6 \mathrm{~N}, 4.6 \mathrm{~N}$, and $7.2 \mathrm{~N}$ in $x, y$, and $z$ direction, respectively. For $25 \mathrm{~kg}$ BWS, the
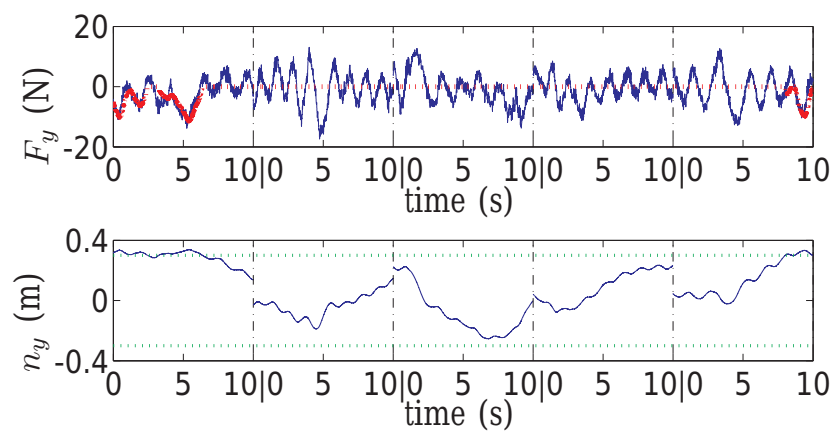

Fig. 7. Concatenated data excerpts from the 5 subjects in $y$-direction Top: Reference force (dotted red) and measured force (solid blue). Bottom: Associated node movement in $y$-direction, with virtual walls (dotted green).
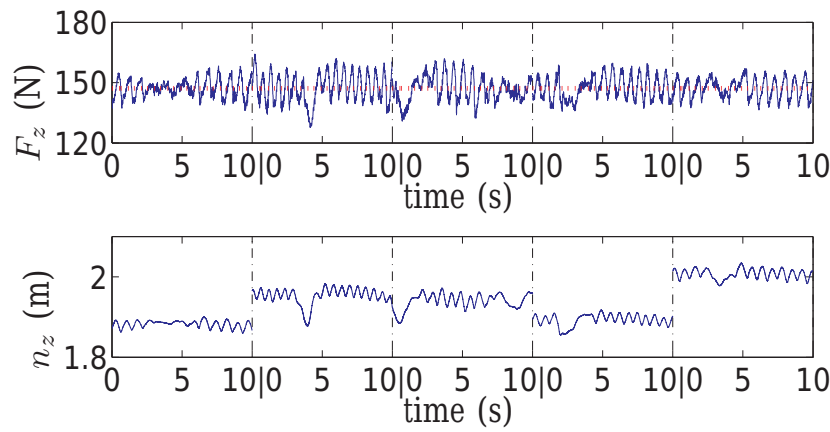

Fig. 8. Concatenated data excerpts from the 5 subjects in $z$-direction. Top: Reference force (dotted red) and measured force (solid blue). Bottom: Associated node movement in $z$-direction.

RMS error in force was $9.7 \mathrm{~N}, 5.7 \mathrm{~N}$, and $9.6 \mathrm{~N}$ in $x, y$, and $z$ direction, respectively.

In the bottom plots of the figures, it can be seen that the movements in the $z$-direction contain small periodic movements of $1-2 \mathrm{~cm}$ amplitude. The position along the $y$-axis varies more, although it also contains a frequency component with an amplitude of $2-3 \mathrm{~cm}$. In the $x$-direction, periodic movements are also present, but they are hidden by the large slopes corresponding to the forward and backward movements. The self-selected walking speed for all subjects was between $1 \mathrm{~m} / \mathrm{s}$ and $1.5 \mathrm{~m} / \mathrm{s}$ in steady state, without visible influence of the unloading force. Speed oscillations in $x$-directions were in the range of about $0.1 \mathrm{~m} / \mathrm{s}$ to $0.2 \mathrm{~m} / \mathrm{s}$ in amplitude.

\section{DISCUSSION}

The achieved force control performance enables highly transparent interaction with walking subjects. This is particularly true for the walking $(x)$-direction, where the passive degrees of freedom, represented by the trolleys, take the function of hiding virtually all inertia of the actuation units.

As a consequence of this transparency, subjects exhibited rhythmic movements in all three directions that were similar in amplitude to what has been reported in literature for free overground gait. However, there are still undesired forces in response to these movements. In the future, we expect this behavior to be further improved once the IMU is integrated in the control scheme. Furthermore, we have already developed 
a non-causal extension to the controller (also presented at this conference [21]), which is based on synchronizing oscillators.

The current controller only applied vertical forces on the subjects, no dedicated assistance in lateral direction was given (besides virtual walls at the workspace limits). For specialized therapy, further algorithms may support subjects in balance tasks, possibly based on the approach we proposed in [22]. The question remains how humans might alter their balance reactions when additional help is provided. The FLOAT offers a research platform to address this question, as well as more general questions on human balance control and falls: In contrast to existing experimental techniques, which induce sudden translations at the feet [23] via perturbation platforms or treadmills, the FLOAT enables perturbations on the upper body during overground locomotion.

\section{CONCLUSION}

In this contribution, we presented the overhead support robot FLOAT, which can apply forces in three directions on the upper body of a human subject. We outlined the control concept and showed first results on force control performance, which demonstrate device transparency. Our next steps are to iteratively evaluate and improve control algorithms in experiments with non-impaired subjects and patients.

In the long run, we aim to make the FLOAT available for diverse therapeutic and research applications: As a therapeutic device, FLOAT can not only support the weight of a user during voluntary movements, but it can also help to initiate movements and to track a 3D reference trajectory for the body's center of mass. Recorded data can also serve diagnostic and prognostic purposes. As a research platform, FLOAT can emulate reduced gravity conditions, but it can also apply horizontal force components on the upper body, for example to laterally perturb or assist the upper body during gait.

\section{ACKNOWLEDGMENT}

The authors would like to thank the Walter L. \& Johanna Wolf-Foundation for financial support, as well as Johann Wanek for help with GUI and electronics, Lea Awai for support during experiments, and Andrew Pennycott for contributions to efficient coding. CE and RR were also supported by the European Community's 7th Framework Programme (FP7/20072013) under Grant 287894 (CYBERLEGs), and the F.R.S.FNRS (1.5025.12 and 2012/V 3/5/394 - IB/JN - 14808).

\section{REFERENCES}

[1] J. L. Emken, R. Benitez, and D. J. Reinkensmeyer, "Human-robot cooperative movement training: Learning a novel sensory motor transformation during walking with robotic assistance-as-needed," Journal of NeuroEngineering and Rehabilitation, vol. 4, no. 8, 2007.

[2] M. A. Guadagnoli and T. D. Lee, "Challenge point: a framework for conceptualizing the effects of various practice conditions in motor learning." J of Motor Behavior, vol. 36, no. 2, pp. 212-224, Jun 2004.

[3] J. Patton, D. A. Brown, M. Peshkin, J. J. Santos-Munné, A. Makhlin, E. Lewis, E. J. Colgate, and D. Schwandt, "KineAssist: design and development of a robotic overground gait and balance therapy device." Top Stroke Rehabil, vol. 15, no. 2, pp. 131-139, 2008.

[4] N. Dominici, U. Keller, H. Vallery, L. Friedli, R. van den Brand, M. L. Starkey, P. Musienko, R. Riener, and G. Courtine, "Versatile robotic interface to evaluate, enable and train locomotion and balance after neuromotor disorders," Nature Medicine, vol. 18, pp. 1142-1147, 2012.
[5] R. van den Brand, J. Heutschi, Q. Barraud, J. DiGiovanna, K. Bartholdi M. Huerlimann, L. Friedli, I. Vollenweider, E. Moraud, S. Duis et al. "Restoring voluntary control of locomotion after paralyzing spinal cord injury," Science, vol. 336, no. 6085, pp. 1182-1185, 2012.

[6] J. Hidler, D. Brennan, I. Black, D. Nichols, K. Brady, and T. Nef, "ZeroG: overground gait and balance training system." $J$ Rehabil Res Dev, vol. 48, no. 4, pp. 287-298, 2011.

[7] A. Pennycott, D. Wyss, H. Vallery, and R. Riener, "Effects of Added Inertia and Body Weight Support on Lateral Balance Control During Walking," in Proc. of the IEEE Int. Conf. on Rehabilitation Robotics (ICORR), Zürich, Switzerland, 2011, pp. 1 -6.

[8] NASA. (accessed on Feb 9, 2013) Active Response Gravity Offload System (ARGOS). [Online]. Available: http://www.nasa.gov/centers/johnson/engineering/integrated_environments/active_response_gravity/index.html

[9] D. Shetty, A. Fast, and C. Campana, "Ambulatory suspension and rehabilitation apparatus," U.S. Patent 7462 138, 2008.

[10] D. Surdilovic, R. Bernhardt, T. Schmidt, and J. Zhang, Advances in rehabilitation robotics : Human-friendly technologies on movement assistance and restoration for people with disabilities. Berlin: Springer, 2004 (Lecture Notes in Control and Information Sciences 306), 2004, ch. STRING-MAN: A novel wire-robot for gait rehabilitation.

[11] T. Bruckmann, "Auslegung und Betrieb redundanter paralleler Seilroboter," Ph.D. dissertation, University Duisburg-Essen, Chair of Mechatronics, 2010.

[12] J. Zitzewitz, L. Fehlberg, T. Bruckmann, and H. Vallery, Cable-Driven Parallel Robots, ser. Mechanisms and Machine Science. Springer Berlin Heidelberg, 2013, vol. 12, ch. Use of Passively Guided Deflection Units and Energy-Storing Elements to Increase the Application Range of Wire Robots, pp. 167-184.

[13] H. Vallery and P. Lutz, "Apparatus for unloading a user's body weight during a physical activity of said user, particularly for gait training of said user," Patent Application EP12 154 778.0, 2012.

[14] S. Fang, Design, Modeling and Motion Control of Tendon Based Parallel Manipulators. VDI-Verl., 2005.

[15] J. von Zitzewitz, G. Rauter, R. Steiner, A. Brunschweiler, and R. Riener, "A versatile wire robot concept as a haptic interface for sport simulation," in Proceedings of the IEEE International Conference on Robotics and Automation (ICRA), 2009, pp. 269-274.

[16] G. A. Pratt, M. M. Williamson, P. Dillworth, J. Pratt, K. Ulland, and A. Wright, "Stiffness Isn't Everything," in International Symposium on Experimental Robotics (ISER), 1995.

[17] H. Vallery, J. Veneman, E. van Asseldonk, R. Ekkelenkamp, M. Buss, and $\mathrm{H}$. van der Kooij, "Compliant Actuation of Rehabilitation Robots - Benefits and Limitations of Series Elastic Actuators," IEEE Robotics \& Automation Magazine, vol. 15, no. 3, pp. 60-69, 2008.

[18] J. von Zitzewitz, G. Rauter, H. Vallery, A. Morger, and R. Riener, "Forward Kinematics of Redundantly Actuated, Tendon-Based Robots," in Proceedings of the IEEE International Conference on Intelligent Robots and Systems (IROS), Taipei, Taiwan, 2010.

[19] J. von Zitzewitz, A. Morger, G. Rauter, L. Marchal-Crespo, F. Crivelli, D. Wyss, T. Bruckmann, and R. Riener, "A reconfigurable, tendon-based haptic interface for research into human-environment interactions," Robotica, 2012.

[20] H. Vallery, R. Ekkelenkamp, H. van der Kooij, and M. Buss, "Passive and Accurate Torque Control of Series Elastic Actuators," in Proceedings of the IEEE International Conference on Intelligent Robots and Systems (IROS), San Diego, USA, 2007, pp. 3534-3538.

[21] C. Everarts, H. Vallery, M. Bolliger, and R. Ronsse, "Adaptive Position Anticipation in a Support Robot for Overground Gait Training Enhances Transparency," in Proceedings of the IEEE International Conference on Rehabilitation Robotics (ICORR), 2013.

[22] H. Vallery, A. Bögel, C. O’Brien, and R. Riener, "Cooperative Control Design for Robot-Assisted Balance during Gait," at-Automatisierungstechnik, vol. 60, no. 11, pp. 715-720, 2012.

[23] H. van der Kooij, E. H. van Asseldonk, J. Geelen, J. P. van Vugt, and B. R. Bloem, "Detecting asymmetries in balance control with system identification: first experimental results from Parkinson patients," $J$ Neural Transm, vol. 114, pp. 1333-1338, 2007. 